\title{
Perinatal and infant postmortem examinations: how well are we doing?
}

\author{
G M Vujanić, P H T Cartlidge, J H Stewart, A J Dawson
}

\begin{abstract}
Aim-To investigate the quality of perinatal and infant necropsies and assess the relation between the quality and value of this investigation in different outcome groups.

Methods-Cohort analysis of 540 deaths during 1993 of babies between 20 weeks' gestation and one year of age born to women usually resident in Wales. Cases were identified from the All Wales Perinatal Survey. Each case was assessed to establish whether the necropsy yielded clinically relevant information. The quality of necropsy was assessed by scoring aspects identified as being part of the necropsy.
\end{abstract}

Results-Necropsy was performed in 335 (62\%) cases, and the report was available for assessment in 314 cases. The quality of necropsy was below the minimum standard in 46\% (143/314) of cases. The highest quality necropsies were carried out on fetal deaths at 20 to 23 weeks' gestation (12\% (10/85) below standard), compared with $65 \%(87 / 133)$ below standard on stillbirths and $68 \%(21 / 31)$ on sudden unexpected infant death. Overall, $42 \%$ (131/314) of necropsies were performed in a regional paediatric pathology centre including $88 \%$ (76/88) of fetal deaths, $23 \%$ (31/133) of stillbirths and $30 \%(29 / 96)$ of infant deaths. The quality score for the necropsy performed in a regional centre failed to achieve the minimum acceptable score in $8 \%$ (11/131) of cases compared with $72 \%$ (132/182) for those done elsewhere. The cause of death was detected by necropsy in $17 \%(52 / 314)$ of cases. The quality of necropsy was significantly higher when the cause of death was revealed than when nothing new was found.

Conclusions-The overall quality of the perinatal and infant necropsy is poor. This is regrettable as valuable information can be revealed frequently by a good quality necropsy. Adherence to Guidelines for Postmortem Reports recently published by the Royal College of Pathologists should improve the situation.

(f Clin Pathol 1995;48:998-1001)

Keywords: Audit, perinatal, infant, necropsy, quality.

The importance of the postmortem examination in identifying the cause of death and as an audit of clinical practice is well established. ${ }^{12}$ Despite this, the necropsy rate in adults has steadily declined from $60-80 \%$ in 1950 to a mere $10 \%$ in $1980 .{ }^{1}$ There is a higher necropsy rate in perinatal medicine, but even here the rate has fallen to about $60 \% .^{34}$ Yet the perinatal necropsy can be especially beneficial as it may also reveal information relevant to future pregnancies and permit more accurate parental counselling. . $^{5-10}$

The reason for the declining postmortem rate is uncertain. Part of the problem seems to be a low motivation among clinicians who may fail to recognise how much clinically relevant information can be obtained from a good quality examination. Some of the fault may lie with pathologists, however, for not addressing clinical questions and performing necropsies of unsatisfactory quality. ${ }^{311}$ The aim of this study was to evaluate the quality of the perinatal and infant postmortem examination in different clinical situations and to assess the relation between the quality and value of this investigation.

\section{Methods}

All 540 deaths during 1993 of babies between 20 weeks' gestation and one year of age born to women usually resident in Wales were included in the study. Cases were identified from the All Wales Perinatal Survey, a population based project which incorporates the Confidential Enquiry into Stillbirths and Deaths in Infancy. Wales covers an area of 8016 square miles and has a total population of nearly three million. There were 36793 births in 1993 and perinatal care was delivered by 16 consultant obstetric units and 11 general practitioner units within Wales, and several hospitals in neighbouring English health authorities. Perinatal and infant pathology services were mainly provided by the local pathologist, although the majority of fetal deaths at 20 to 23 weeks' gestation were examined in a regional paediatric pathology centre.

Information about each case was collected on the survey form which was normally completed by the team in whose unit the baby died. This included clinical details about the mother's medical and obstetric history, pregnancy, labour, and delivery. Data about the baby included sex, birth weight, gestational age, clinical management, and a copy of the postmortem report.

The cases were divided into six groups: spontaneous abortions at 20 to 23 weeks' gestation, therapeutic abortions at 20 to 23 weeks' gestation, fresh stillbirths, macerated stillbirths, 
Table 1 Scoring system for the quality of the perinatal and infant necropsy

\begin{tabular}{lc}
\hline Category & Score \\
\hline Body weight & 20 \\
Crown rump/heel length & 20 \\
Head circumference & 20 \\
Foot length & 20 \\
Assessment of gestational age & 20 \\
Organ weights-main* & 40 \\
Organ weights_other & 40 \\
Normal values (for organ weights) & 20 \\
Histology—-main organs & 50 \\
Histology_other organs & 50 \\
Placenta-macroscopy & 50 \\
Placenta-microscopy & 50 \\
Radiology & 100 \\
Microbiology & 100 \\
Other relevant investigations & 100 \\
(e.g. cytogenetics, biochemistry) & \\
\hline
\end{tabular}

* Main organs are brain, liver, lungs, and kidneys.

sudden unexplained infant death (SUID), and other infant deaths (non-SUID). The quality of each necropsy report was assessed using a modification of the system described by Rushton, ${ }^{12}$ which objectively scores aspects identified by the Royal College of Pathologists as being part of a necropsy (table 1 ).${ }^{13}$ Six aspects of the infant necropsy (body measurements, organ weights, histology, radiology, microbiology, other relevant investigations) were assessed giving a maximum score of 600 . The necropsy after a fetal death (late abortions and stillbirths) also included an examination of the placenta so the maximum score was 700 . The minimum acceptable score was also based on the Royal College of Pathologists guidelines, and for the fetal necropsy it was set at 350 (body measurements, organ weights, main organ histology, and examination of the placenta). The infant necropsy does not include examination of the placenta and so the minimum acceptable score for non-SUID cases was 250. The necropsy for SUID cases needs to be more detailed and so the minimum acceptable score was set at 350 .

To evaluate the benefit of the necropsy, each case was assessed to determine what new information was revealed by the examination. The clinical details alone were assessed by a panel consisting of a neonatal paediatrician, an obstetrician, a paediatric pathologist, and a midwife. The panel then reassessed each case using the findings from the necropsy to evaluate how informative the necropsy had been. The following system was used: diagnostic, new information which revealed the primary cause of death or which had important implications for future pregnancies; additional, new information but not diagnostic; audit, information useful for clinical audit; none, no new information and not useful for audit.

\section{Results}

A postmortem examination was performed in $62 \%(335 / 540)$ of cases. Twenty one necropsy reports were unavailable for assessment, so 314 cases were used in this analysis. Scores for the quality of the 314 necropsy reports ranged from 0 to 700 , with a mean of 306 . There were 85 fetal deaths at 20 to 23 weeks' gestation (spontaneous and therapeutic abortions), with a mean score (95\% confidence interval (CI)) of 419 (95\% CI 397 to 440 ). By comparison, the mean score for 133 stillbirths was 263 (95\% CI 237 to 288). In 65 non-SUID cases the mean score was 264 (95\% CI 240 to 289) and in 31 SUID cases it was 265 (95\% CI 213 to 315 ).

Scores for the quality of the necropsy report failed to attain the minimum acceptable score in $46 \%(143 / 314)$ of cases. The highest quality reports were for fetal deaths at 20 to 23 weeks' gestation (late abortions) with only $12 \%$ (10/ 85) falling below the minimum acceptable score of 350 (table 2). By comparison, the quality score for necropsies of stillbirths (fresh and macerated) failed to attain the minimum acceptable score of 350 in $65 \%(87 / 133)$ of cases; in $46 \%(61 / 133)$ the score was less than 250 and in $32 \%(42 / 133)$ it was less than 150 . There were 96 infant deaths and in $48 \%$ (46 cases) the necropsy report failed to reach the minimum acceptable score. In non-SUID cases $38 \%(25 / 65)$ were below the minimum acceptable score of 250 , and in $11 \%(7 / 65)$ it was below 150 . In SUID cases $68 \%(21 / 31)$ of reports failed to reach the minimum acceptable score of 350 ; in $45 \%$ of cases (14/31) it was less than 250 and in $23 \%(7 / 31)$ it was below 150.

Diagnostic information was found in $17 \%$ (52/314) of cases, additional information in $9 \%$ (27 cases), audit information in $11 \%$ (36 cases) and no new information in $63 \%$ (199 cases). In cases in which the necropsy yielded diagnostic information the mean score (95\% CI) was 349 (95\% CI 312 to 386), compared with 289 (95\% CI 268 to 310 ) in cases where no new information was found (95\% CI for difference between scores 14 to 106). A similar trend was seen in individual groups (abortions, stillbirths and non-SUID cases), although the difference was only statistically significant for fresh stillbirths (table 2).

Overall, $42 \%$ (131/314) of necropsies were performed in a regional paediatric pathology centre including $76 / 86(88 \%)$ fetal deaths at 20 to 23 weeks' gestation, $31 / 133$ (23\%) stillbirths and $29 / 96(30 \%)$ infant deaths. The mean score for necropsies performed in a regional centre was 414 (95\% CI 399 to 430$)$ compared with 228 (95\% CI 210 to 246 ) for necropsies per-

Table 2 Quality scores for perinatal and infant postmortem examinations

\begin{tabular}{|c|c|c|c|c|}
\hline \multirow[b]{2}{*}{ Outcome groups } & \multirow{2}{*}{$\begin{array}{l}\text { Mean score } \\
\text { (range) }\end{array}$} & \multirow{2}{*}{$\begin{array}{l}\text { Number of necropsies below } \\
\text { minimum acceptable score (\%) }\end{array}$} & \multicolumn{2}{|c|}{ Score according to the information revealed at necropsy (mean ( $95 \% \mathrm{CI})$ ) } \\
\hline & & & Diagnostic & None \\
\hline $\begin{array}{l}\text { Spontaneous abortions }(n=45) \\
\text { Therapeutic abortions }(n=40) \\
\text { Macerated stillbirths }(n=96) \\
\text { Fresh stillbirths }(n=37) \\
\text { Non-SUID }(n=65) \\
\text { SUID }(n=31)\end{array}$ & $\begin{array}{l}410(90-600) \\
429(150-600) \\
275(0-700) \\
231(20-580) \\
264(60-500) \\
265(0-580)\end{array}$ & $\begin{array}{l}4(9 \%) \\
6(15 \%) \\
58(60 \%) \\
29(78 \%) \\
25(38 \%) \\
21(68 \%)\end{array}$ & $\begin{array}{l}449(417-480)(n=16) \\
500(n=1) \\
304(220-389)(n=14) \\
410(249-571)(n=4) \\
277(225-328)(n=13) \\
245(147-343)(n=4)\end{array}$ & $\begin{array}{l}398(356-441)(n=24) \\
455(413-498)(n=22) \\
262(228-295)(n=77) \\
190(151-229)(n=24) \\
232(187-277)(n=25) \\
267(210-325)(n=27)\end{array}$ \\
\hline
\end{tabular}


formed elsewhere. The quality score for the necropsy performed in a regional centre failed to achieve the minimum acceptable score in $8 \%(11 / 131)$ of cases, compared with $72 \%$ $(132 / 182)$ for those done elsewhere.

\section{Discussion}

The perinatal and infant necropsy rate in Wales for 1993 was only $62 \%$, well below the minimum target of $75 \%$ set by a joint working party of the Royal College of Pathologists and Royal College of Obstetricians and Gynaecologists. ${ }^{14}$ However, it is likely that this rate is typical of the rest of Britain; in several regions it is below $50 \%$ ( $R$ Stanwell-Smith, personal communication). ${ }^{415}$

Overall, the quality of perinatal and infant necropsies in Wales was below the desired standard in $46 \%$ of cases. In two comparable reviews of perinatal cases in the West Midlands and in East Anglia, similar results have been found with, respectively, $44 \%$ and $48.3 \%$ of necropsies below standard. ${ }^{412}$ These authors used a similar method of assessment to that used in our study, with the quality of the necropsy report rather than the examination being evaluated. Nevertheless, we believe that this method gives an acceptable means of assessing the quality of the postmortem investigation.

In our study the quality of necropsies varied considerably according to the timing of the death. The highest quality was seen in therapeutic and spontaneous abortions, with only $12 \%$ of reports failing to achieve the minimum acceptable score; the majority of these cases were examined in a regional paediatric pathology centre. By comparison, in $65 \%$ of stillbirths the necropsy was below standard, which suggests that a careful postmortem examination is perceived as a low priority in this group. This perception would not be supported by our data as in our study the postmortem examination revealed the cause of death in $14 \%$ of stillbirths. Furthermore, we have confirmed our previous finding that in perinatal and infant deaths in general, ${ }^{5}$ and in fresh stillbirths in particular, the cause of death is significantly more likely to be revealed by a high quality postmortem examination. This supports the findings of Porter and Keeling ${ }^{6}$ who reported that in $60 \%$ of necropsies performed by a paediatric pathologist, new information was revealed by the examination, and that in $20 \%$ histological examination led to the correct diagnosis. The importance of the quality of the necropsy is emphasised by the finding that the quality score was significantly higher in those cases where new diagnostic information was obtained than in cases where no new information was found.

The quality of necropsies in infant deaths has not been reported previously. In $48 \%$ of these cases the postmortem examination failed to achieve the minimum acceptable score. The lowest quality was in SUID cases where $68 \%$ were below standard. This is perhaps the most disturbing of our findings as, by definition, sudden infant death syndrome (SIDS) is "the sudden death of an infant or young child which is unexpected by history and in whom a thor- ough postmortem examination fails to demonstrate an adequate cause of death". ${ }^{16}$ It follows that in infants dying suddenly, a good quality postmortem examination is essential before the diagnosis of SIDS can be made. If the incidence of SIDS continues to decline pathologists will be faced with a higher proportion of explicable SUID cases which will increase their responsibility in reaching a final diagnosis. ${ }^{17}$

Our study confirms the value of the perinatal and infant postmortem examination. Clinically relevant information was revealed by necropsy in $17 \%$ of cases, giving clinicians and parents a better understanding of the cause of death and providing a basis for informed genetic counselling. Moreover, in $11 \%$ of cases information useful for auditing new diagnostic and therapeutic techniques was found. Previous reports have described similar benefits, with clinically important information uncovered at necropsy in 14 to $46 \%$ of cases. ${ }^{5611}$ Even in cases where no new information is found at necropsy, it is reassuring for parents and clinicians to know that important disease has not been missed. However, such reassurance can only be given if the necropsy is of good quality; Rushton pointed out that "the badly performed necropsy is probably worse than no necropsy at all since it may lead to a false sense of security for the parents". ${ }^{12}$ This is the first study to document a difference between the quality of perinatal and infant necropsies carried out in district general hospitals and regional centres. The reasons for this difference were not determined, but probably include referral of complex cases requiring more detailed examination, and access to techniques and facilities in regional centres not routinely available in district general hospitals. Whatever the reasons, this difference should encourage referral of difficult cases to regional centres for postmortem examination.

This study has shown that the quality of perinatal and infant postmortem examinations is unsatisfactory. This is regrettable as valuable information can frequently be revealed by a good quality necropsy. Although previously published guidelines on performing postmortem examinations ${ }^{1819}$ had little impact, it is hoped that the Guidelines for Postmortem Reports, recently published by the Royal College of Pathologists, ${ }^{13}$ will improve the situation and help to reverse, or at least halt, the declining necropsy rate.

We thank local convenors, district coordinators and pathologists for their help with data collection, and Professor D P Davies, Professor G T Williams and Professor P J Bercy D P Davies, the man $G$ W. We als and Professor $P$ Jerry for reviewing the mey ascript. We also wish to thank Mrs J Mopkins, the Survey administrator, for her support. The All Wales Perinatal Surve incorill

1 Harrison M, Hourihane DO'B. Quality assurance programme for necropsies. 7 Clin Pathol 1989;42:1190-3.

Goldman L, Sayson R, Robbins S, Cohn LH, Bettmann $M$, Weisberg $M$. The value of the necropsy in three medical $\mathrm{M}$, Weisberg $\mathrm{M}$. The value of the necrop
eras. $N$ Engl $₹ \mathrm{Med}$ 1983;308:1000-5.

3 Favara BE, Cottreau C, McIntyre L, Valdes-Dapena M. Favara BE, Cottreau C, McIntyre L, Valdes-Dapena M.
Pediatric pathology and the necropsy. Pediatr Pathol 1989; 9:109-16.

4 Audit of perinatal pathology. East Anglian Regional Perinatal Survey and Confidential Enquiry into Stillbirths and Deaths in Infancy (CESDI). Annual Report, 1993:62-7. 5 Cartlidge PHT, Dawson AJ, Stewart JH, Vujanic GM. 
Value and quality of perinatal and infant postmortem examination: coh

6 Porter HJ, Keeling JW. Value of perinatal necropsy examination. 7 Clin Pathol 1987;40:180-4.

7 Shen-Schwarz S, Neish C, Hill LM. Antenatal ultrasound for fetal anomalies. Importance of perinatal necropsy.

8 Manchester DK, Shikes RH. The perinatal necropsy: special considerations. Clin Obstet Gynecol 1983;23:1125-34.

9 Meir PR, Manchester DK, Shikes RH, Clewell WH, Stewart M. Perinatal necropsy: its clinical value. Obstet Gynecol $1986 ; 67: 349-51$

10 Clayton-Smith J, Farndon PA, McKeown C, Donai D. Examination of fetuses after induced abortion for fetal abnormality. BMF 1990;300:295-7.

1 Schned AR, Mogielnicki P, Stauffer ME. A comprehensive quality assessment program on the necropsy service. $A m$ quality assessment program

12 Rushton DI. West Midlands perinatal mortality survey,
1987. An audit of 300 perinatal autopsies. Br $\mathcal{F}$ Obstet Gynaecol 1991;98:624-7.

13 Royal College of Pathologists. Guidelines for post mortem reports. London: Royal College of Pathologists, 1993.

14 Joint Working Party, RCOG/RCPath. Report on Fetal and Perinatal Pathology. London: Royal College of ObstetPerinatal Pathology. London: Royal

15 Keeling JW, Gibson AAM, Cole SK. Perinatal necropsies in Scotland in 1991 [abstract]. $\mathcal{F}$ Pathol 1994;172(Suppl): A222.

16 Chiswick M. Perinatal and infant postmortem examination. BMF 1995;310:141-2.

17 Valdes-Dapena M. A pathologist's perspective on the sudden infant death syndrome-1991. Pathol Ann 1992;27:133-

18 Langley FA. The perinatal postmortem examination. $f$ Clin Pathol 1971;24:159-69.

19 National Advisory Board for the Confidential Enquiry into Stillbirths and Deaths in Infancy (CESDI). Postmortem Stillbirths and Deaths in Infancy (CESDI). Postmortem
guidelines. National Advisory Board for CESDI, 1993. 